Another important provision of the law enables the Beard of Regents to inquire into the identity of any person claiming to be a licensed physician. No such power was given in the old law. This will make it difficult for a person to practice on the license of a deceased physician, and will render it far easier to detect fraud in medical companies. It will also render it more difficult for quacks to combine and shelter each other, or hide behind licensed practitioners, as they have frequently done in the past. Prosecutions may now be conducted by the society under five sections of the penal code, instead of one as formerly, and also under the special provisions of the new law. The restrictions of the old law making it impossible for a judge to impose a sentence of both fine and imprisonment for a first offense for the illegal practice of medicine has been abolished, so that much heavier and more deterrent penalties may now be imposed.

For several years the volume of legal work has annually increased, and during the year now closing it has exceeded in every particular that of any former year. The number of prosecutions, convictions, fines, and imprisonments, and the aggregate amount of fines have all been greater than in any previous year. The work has not only been an evolution in principles and methods, but has yearly expanded in scope and volume.

In the prosecution of this work all sorts and conditions of men have been revealed as doers of evil, some of whom have posed as moral citizens; many lucrative but corrupt business concerns have been disrupted; many influential citizens and powerful interests have been hard hit. As the work has broadened and the number of such cases has increased, violent animosities have been engendered, not alone against individuals, but against the work itself. And so long as the work is aggressive and effective, opposition to it must be at times expected. To those who feel that the society should limit itself to scientific and professional work. I would call attention to the fact that legal work was one of the essential objects for which it was organized. The title of the legislative act which called this society into existence in 1806, read as follows: "An act to incorporate medical societies for the purpose of regulating the practice of physic and surgery in this state;" and this is incorporated into the constitution as a prime object of the society to-rlay. With such a mandate from the state and in view of the results acromplished, it seems to me that the society should not shirk its dutv as regards the legal work, for it has been a great work, it has been a good work, it has been a clean work.

\section{THE INDICATIONS FOR OPERATION IN ELECTIVE SURGERY.*}

\author{
GERRY R. HOLDEN, M.D. \\ Gynecologist to St. Luke's Hospital. \\ JACKSONVIILE, FLA.
}

Before the use of anesthetics and before the introduction of aseptic principles, major surgery was limited almost entirely to emergency or imperative surgery. That is, operative procedures were employed only when severe, urgent symptoms could be relieved without too great risk from the operation itself, or when an immediate operation was imperatively indicated in order to save life.

* Read at the Thirty-fourth Annual Session of the Florida Medical Association, Tampa, April 17, 1907.
Formerly most operations were in themselves dangerous. Now, in the majority of cases, the risk from the operation itself is slight. The element of danger usually depends on the severity of the condition which makes the operation necessary. This reduction of the operative risk, together with the assurance which we have of obtaining good aseptic results in the great majority of cases, has created for us an entirely new surgical field, that of elective surgery.

The operations of elective surgery are those operations which are usually done in order to relieve distressing symptoms, more rarely in order ultimately to save life. Their especial characteristics are that the operation docs not necessarily have to be done immediately, and that there may often be a question as to whether or not the operation really is advisable.

I will mention a few of the many conditions which may come into this field in order to make the conception of elective surgery more clear. Gynecology offers many instances. Displacements of the uterus, tears of the perneum or cervix, inflamed tubes or ovaries, and many cases of ovarian or uterine tumors are frequent examples. The great majority of such cases do not require immediate operation. Many of these patients do not need an operation in order to save life, and in others the question may be fairly raised as to whether or not it is advisable to do any operation at all.

In general abdominal surgery we may consider nonstrangulated hernia, floating kidney, many cases of gallstone or gastric ulcer, and suspected chronic appendicitis which has never shown any symptoms of acute attacks. Without extending this list further, I think enough conditions have been given to impart a fairly clear idea of the sort of case coming in the province of elective surgery.

In imperative surgery the diagnosis is often clear and the necessity for an immediate operation is usually apparent. In such cases there is but one major responsibility devolving on the physician. That is the responsibility of having a sufficiently skillful and satisfactory operation performed at once.

In elective surgery, however, there are usually two major responsibilities. There is as great a need for a skillful operation as in imperative surgery, but of equal, if not of greater importance, is the responsibility of first deciding whether or not the paticnt actually needs any operation at all.

Who is the man to settle this question? The operator assumes the moral and professional responsibility for the operation. His judgment dictates the procedures used; his is the actual credit if the operation is successful, and his is the blame if it is not. Inasmuch as the operator will thus have to bear the responsibility for the results of the proposed operation, it is only fair that he be allowed to decide whether or not operation is necessary.

To aid the surgeon to make a wise decision he must be given every opportunity for full and free examination of the patient. He should be permitted to express his opinion freely, and should be allowed to follow unhampered the dictates of his own best judgment. The surgeon who waives this privilege of prior examination, unless compelled thereto by extreme circumstances, who sees the patient for the first time after anesthesia has begun, and who does a certain routine operation at another's request, degrades himself and his profession. He assumes the status of a skilled mechanic rather than that of the scientifie physician. His operation is human 
carpenter work, done to order, rather than scientific surgery.

On the other hand, we should not tie the surgeon's hands and hinder him in his attempt to do his best work by sending patients to him with the request that he perform a certain specified operation. Rather let us tell the patient that an operation may be necessary, but, inasmuch as the surgeon has to stand for the results of his operation, let us permit the surgeon himself to decide whether or not an operation will be necessary in a given case of elective surgery.

It is manifestly impossible to go over all conditions and operations coming at times under the heading of elective surgery. I do wish, though, to take up a few special procedures and consider them in some detail from the standpoint of operative indications.

From the particular points which this study will bring up I hope that we shall be able to deduce conclusions applicable to the whole field. As most gynecologic conditions belong to elective surgery, so most of the operations of gynecology come under this heading, and I shall confine my consideration of special operations to this line.

\section{DILATATION AND CURETTAGE.}

Probably the gynecologic operation which is done more frequently than any other is dilatation of the cervix uteri, followed by curettage of the endometrium. While in many cases the operation is indicated and the good results justify it, yet I have no hesitation in stating my belief that a large percentage of all these operations are unnecessary, unjustifiable, and frequently actually injurious.

The majority of all curettages are done on account of one of four or five reasons: (1) As treatment for endometritis or general inflammatory conditions of the pelvic organs; $(2)$ as treatment for dysmenorrhea or sterility; (3) as treatment for, or diagnosis of, uterine hemorrhage; (4) empirically, in poorly understood, obstinate cases, with the hope that in some unknown way good results may follow.

Curettage in Inflammatory Conditions.-Very careful examination is necessary before resorting to the curette in actual inflammatory processes of the endometrium. In acute endometritis the consensus of opinion is that curettage is absolutely contraindicated if there be any involvement of the tubes, and, even when the tubes are free from infection, it is usually inadvisable. Curettage in acute endometritis usually increases the degree of inflammation and leaves the patient's last condition worse than the first.

In chronic endometritis the operation may be of great benefit, but care is needed in selecting cases. Whenever there is any suspicion of tubal infection the operation is usually contraindicated.

I will merely mention in passing that actual chronic endometritis, that is, a true chronic inflammation from the pathologic standpoint, is not often seen without accompanying inflammatory changes in the tubes also. The clinical symptoms which are usually considered those of chronic endometritis are actually due to a numbor of different pathologic conditions of the endonetrium, most of which are not at all inflammatory in or: $\sin$.

Curettage in Dysmcnorrhea or Sterility.-This operation is frequently employed in treating dysmenorrhea or sterility. Though dilatation for dysmenorrhea was originally based on the theory that an obstruction in the menstrual flow was the cause of one form of dysmenor rhea, the procedure has since been applied to every form of dysmenorrheal pain. A few years ago I made a study of the results of this treatment. ${ }^{1}$ I was able to get reports from nearly one hundred wornen at periods varying from one to twelve years after curettage for dysmenorrhea had been done at the Johns Hopkins Hospital. This study showed that the operation is far more likely to give good results in those patients whose monthly pains are sharp or paroxysmal, begin on the day of flow or the day before, and last but a day or two. The likelihood of relief is much less when the pain is dull in character, begins several days before the flow appears, and lasts for several days or throughout the flow.

Before dilating and curetting for dysmenorrhea we must satisfy ourselves that the symptom is not due to some general or local pathologic condition. Manifestly dilatation of the cervix would do no good in dysmenorrhea caused by a retroflexed uterus or an inflamed tube; in like manner dysmenorrhea in a neurasthenic patient is not likely to be relieved unless the neurasthenia is also relieved.

A word as to the value of this operation in relieving sterility. While sometimes women, previously sterile, do become pregnant so soon after a dilatation that it seems as if the operation must have relieved the sterility, yet as a routine operation I do not believe that it will give a large percentage of successful results. In my study on dysmenorrhea, just referred to, there were twenty married women who had been sterile before the dilatation and curettage. Of these twenty women but three became pregnant within six months after the operation. It bchooves us, therefore, to be very chary in promising results from dilatation as a method of curing sterility.

Dilatation and Curettage in Uterine Hemorrhage.In the diagnosis and treatment of uterine hemorrhage curettage reaches its noblest and most useful plane. In every case of uterine hemorrhage, when the cause of the bleeding can not be satisfactorily explaines by the findings at the ordinary pelvic examination, a thorough curettage should be done and the curettings examined microscopically by a skilled pathologist. In this way we may frequently diagnose an early case of cancer of the uterus when, with the exception of bleeding, all symptoms of cancer are lacking. Since the meeting of last year I have been able to diagnose two very early cases of cancer by this method. Neither of the women had any symptoms of cancer except an irregular uterine bleeding, which local examination dir not explain satisfactorily. Each patient was curetted and the pathologic examination of each specimen showed cancer. A thorough operation was done in each case and I feel that we have good assurance tlat the life of each patient will be saved. If so, it will be due in great part to the diagnosis given us by the curettage.

As a treatment for uterine hemorrhage the curette is most successful in cases of retained secundines after labor or miscarriage and in the so-called chronic polypoid endometritis. When the bleeding comes from one or more large uterine polypi the curette may be sufficient, but it is often hard to remove these tumors by curetting alone. Curettage will check the bleeding in acute or chronic endometritis, but from the considerations given above it should be done only as a last resort in such cases.

\footnotetext{
1. American Medicine, Nov. 4, 1905.
} 
Before performing curettage in any case of uterine hemorrhage it is necessary to rule out the presence of an extrauterine pregnancy. Contrary to what we might gather from most text-books, extrauterine pregnancy does not usually have for its first symptoms those of shock from the uncontrolled bleeding of a ruptured tube. Out of eighteen patients on whom I have operated for extrauterine pregnancy during the last two years but two had the classical text-book symptoms. The others were all able to walk either to my office or to the hospital. Irregular uterine hemorrhage was a symptom in each of these cases. Currettage in a case of extrauterine pregnancy would be a most unreasonable procedure, certain to do no good, ard very likely to do great harm.

Curettage from Empirical Reasons.-Finally, I am sorry to say, curettage is sometimes done in an unreasoning, empirical way, with the hope that in some mysterious manner a patient who has heretofore resisted all treatment may be benefited. In such instances the case is usually poorly understood, no accurate diagnosis is made, and the operation is done with no definite idea as to how it is to produce the desired results. If one should ask the operator what his justification for the operation was he would probably reply that he had tried everything else he knew, that this would do the woman no harm and that it might do her good.

of course such operating is not surgery. The conscientious surgeon always remembers that there is a certain element of danger in every operation, no matter how trivial the procedure may be. He never advises an operation unless he feels assured that it is definitely indicated in the case in question.

\section{OPERATION FOR CERVICAL LACERATION.}

The procedures which I wish to consider next are those intended to repair the lacerated cervix.

A certain degree of laceration of the cervix must be regarded as an invariable result of every labor. In most cases these wounds of the cervix heal kindly, with no infection, and leave a good scar. Some ilegree of healed cervical laceration, therefore, must be regarded as practically a normal condition in every woman who has borne children.

Operation is sometimes necessary in old cervical lacerations for one of two reasons. First, on account of the local inflammation about an unhealed tear; and, second, as a prophylactic measure against cancer of the cervix.

A cervical laceration, with engorged, congested surfaces, the dilated glands pouring forth a profuse mucoid secretion, certainly calls for operation when local treatment fails to relieve the condition.

Operating on a well-healed scar of the cervix with the view of preventing a possible occurrence of cancer in the future is, however, a question for careful consideration. Most cervical cancers do start in old lacerations, it is true. That is no justification, however, for repairing every laceration, as the separate individual's chance of having a cancer is very slight. Some lacerations are so extensive, though, even when well healed, that, coupled with a family history of cancer, we do sometimes seem justified in repairing them even merely as a prophylactic measure.

Looking at the subject of cervical laceration in general, it must be confessed that not all the operations done are justified or necessary. There are two main paths of error which may lead the ambitious operator to the performance of needless cervical repairs. The first lies in the old belief that varions hysterical and neurotic symptoms may arise as reflex phenomena from a lacerated cervix. ' $\mathrm{T} l \mathrm{l}^{2}$ second lies in the fact that the surgeon may fail to discover the diseased conditions of tube or ovary, which is the real cause of the symptoms, and attribute all the trouble to the cervical laceration which he easily makes out.

The idea that general nervous conditions can arise directly as reflex phenomena from lacerations of the cerrix, or, indeed, from any other diseased condition of the pelvic organs, is now being abandoned by nearly all prominent neurologists and many gynecologists. Neurasthenia and hysteria, when associated with gynecologic troubles, either exist independently of them or else are merely the symptoms of the final nervous breakdown which any form of long continued pain and suffering may cause in women of naturally neurotic temperament. Healed scars of the uterine cervix can cause reflex neurasthenia or hysteria no more than can a well-healed wound on the leg or arm.

The second reason which may lead one to do unnecessary cervical operations is, as I have said, the failure to discover the pelvic lesion which is the real cause of the trouble. Such a mistake may seem at times almost excusable. Laceration of the cervix is one of the easiest pathologic conditions in all medicine to diagnose. Many other patho'ogic conditions of the pelvic organs are very hard to make out, especially when the patient is not under an anesthetic. What then is more natural for the man who has not a wide experience in gynecology and who, perhaps, does not appreciate keenly the proper relation between pathologic conditions and subjective symptoms, than to miss the inflamed tube and ovary above and attribute his patient's complaints to the innocent, healed scar of the cervix?

The way to avoid these mistakes is, of course, to use the utmost care in ruling out every other condition before ascribing to the laceration the patient's symptoms.

\section{OPERATION FOR UTERINE DISPLACEMENTS.}

The normal position of the uterus is that of slight anteflexion and anteversion, the fundus resting on the bladder, the cervix pointing toward the lower part of the sacrum. Theoretically, any deviation from this position may be regarded as abnormal and as offering a possibility for operative interference. Shall we, therefore, operate on all uteri whose position is in any way different from that which we call "normal"? It seems almost as if this has been the position which has been taken from time to time by different operators. If a woman with a retrodisplaced uterus was unlucky enough to fall into the hands of one of them, operation was immediately advised, no matter whether her symptoms were referable to the displacement or not. A much more sane view is now generally held.

Let us first discuss anteversion and anteflexion. It is now considered that the uterus may have a wide degree of anteflexion or anteversion without really being abnormal at all. When the condition is extreme we usually find that we are really dealing with mal-developed pelvic organs. This maldevelopment, rather than the anteflexion, is the main feature and is no indication for operation. On account of the general acceptance of this view operations for anterior deviations of the uterus are now done much less frequently than formerly.

With retrodisplacements the question is different. ATthough some eminent gynecologists deny that a simple retrodisplacement can in itself cause symptoms, the 
majority do not agree with this view. While we know that in many instances retrodisplacement can exist with no symptoms whatsoever, yet most gynecologists are convinced, by the accumulated mass of clinical evidence, that in other cases the mere displacement does in itself cause distressing subjective symptoms.

An especially careful analysis of symptoms is necessary before advising treatment in this condition. In the case of a hysterical woman with a retrodisplaced uterus it is often difficult to decide whether or not an operation is necessary. There are many hysterical women in whom the uterus is in normal position. On the other hand, many a woman with retrodisplaced uterus is perfectly well. How careful, therefore, must we be before advising operation for this condition in a patient whose predominant symptom is hysteria. If, by careful study of the history, we find that the neurotic symptoms came as a sequel to a long period of pelvic symptoms which were at first free from any hysterical complication, then we can feel that correction of the displacement may do some good. If the woman is first relieved from the chronic, dragging pain, which by its constant strain has finally broken down a naturally weak nerrous srstem, we are more likely to treat the hysteria successfully by medinal means.

Treatment directed toward a retrodisplaced uterus in a case of pure hysteria which has never shown any simptoms directly referable to the displacement is unjustifiable. The good results sometimes obtained in such cases are really the effect of suggestive therapeutics and surgical operations are not justifiable methods of hrpnotisn.

In general, operative interference is indicated in the treatment of retrodisplaced uteri only when local treatment has failed to relieve symptoms directly referable to the displacement. Such symptoms are more frequently met with in multipara than in nulliparx. The displacement then is usually associated with torn perineal muscles and a certain degree of uterine prolapse. The symptoms which most frequently call for treatment are sensations of weight and discomfort in the pelvis, backache and pain in the lower abdomen, often running to the legs. These symptoms are usually intensified by exertion or at the menstrual period. Actual dysmenorrhea is far more frequent in nulliparæ than in multiparæ.

In nullipare we must always be on our guard against a retrodisplacement, which is merely one manifestation of a general maldevelopment of the pelvic organs. Often the anterior wall of the vagina is so short that the uterus is of necessity kept in the posterior position. Operations in such cases are not indicated, as they do nothing to relieve the main factor of the trouble, the maldevelopment.

\section{REPAIR OF THE LACERATED PERINEUM.}

While there are few routine procedures which have brought more relief from suffering to thousands of women than the repair of old perineal lacerations, still the surgeon must always be on his guard to see that the necessary indications are satisficd before advising operation. The mere existence of a tear, even if it extends. completely into the bowel, does not necessarily justify an operation. Many women go for years with the sphincter ani completely torn thrown and yet have a wonderful degree of control wit!. no srinptoms whatsoever from the uterine prolar,se $I n$ surt zases we must assure ourselves that the gennral condition is perfectly good before we are warrantel ip solying that operation is indicated. Operation is calıed for more on account of the severity of the symptoms which the lesion causes rather than the degree of the lesion itself.

In an old laceration of the perineum in which the sphincter ani is not involved the indications are very definite. The most important symptom is the sense of loss of support for the pelvic organs; the patient describes it as feeling that "everything is falling out" of her. In addition we have dragging sensations, pain in the back, more or less abdominal pain, and resultant general physical depression. The pathologic condition which causes these symptoms is the descensus allowed by the weakened pelvic floor below: The operation indicated is one which will restore this muscular floor. Mere suturing of vaginal connective tissues or skin is not sufficient: An operative technic which I described in a previous article ${ }^{2}$ is, I think, about the most satisfactory and simple method of accomplishing this result.

\section{OÖPHORECTOMY.}

The history of oöphorectomy presents the same features that we find common to many other operations, capable of accomplishing much good. After its value was finally demonstrated the medical world ran wild for a time; oöphorectomy was done in every sort of condition and hundreds of women were needlessly mutilated. The inevitable reaction came later and now we realize that, while this operation is a procedure of greatest value in its proper place, reckless oöphorectomy, without proper indications, can do an enormous amoun't of damage.

To remove both ovaries is an operation of the greatest magnitude, not on account of any difficulty in the operative technic, but because of the serious after-effects which it may bring to the patient. The nervous and mental disturbances which the premature removal of both ovaries sometimes occasions is often much worse than the original symptoms for the relief of which the operation was performed.

The following conditions will cover most of the indications for oöphorectomy: Malignant disease of the uterus, ovarian cysts or tumors, some cases of pelvic inflammatory disease and some cases of myomata.

One important point, from the patient's standpoint, is whether or not one ovary or a part of one is left in situ. If it is possible to do this the nervous symptoms which follow after the entire removal of both ovaries do not occur. The theory is that the ovary has an internal secretion poured into the blood, which exerts a powerful effect on the general nervous system. One ovary, or even a part of one ovary, can keep up this internal secretion and thus spare the patient the poss bility of many distressing symptoms.

With these points in mind, let us examine the conditions which I have just mentioned as possible indications for oöphorectomy.

In malignant disease of the uterus conservatism is thrown to the winds, and all the pelvic organs, including both ovaries, should be removed. Malignant disease demands most heroic treatment if other than palliative result is desired.

The indication for operation in orarian ersts or tumors lies in the presence of the tumor itself. Every ovarian tumor should be removed as soon as possible after diagnosis is made unless disease of some other organ renders an operation especially dangerous. Early interference is indicated for a number of reasons, but especially because malignant disease of the ovary is fre-

2. The Amer. Jour. of Obstet,, October, 1905 . 
quent, and because it is often impossible to tell with certainty by local examination whether an ovarian tumor is malignant or not. Neither extreme youth nor old age are in themselves contraindications to the removal of ovarian tumors. Old women usually stand this operation remarkably well, while in little girls malignant disease of the ovary is by no means uncommon.

The ovarian tumor is usually unilateral, and whenever possible the other ovary should be left.

The indication for oöphorectomy in inflammatory troubles depends solely on the pathologic condition of the ovaries, not on the patient's cymptoms. The focus of infection is the tube in most cases. The oöphoritis then begins at the outside of the ovary and oftentimes completely clears up on removal of the offending tube. The indications for oöphorectomy in inflammatory diseases of the pelvic organs are, therefore, about as follows: When the tube and ovary are so bound together by adhesions that removal of the tube alone is technically impossible, the ovary must also be removed. If both ovaries are in this condition, efforts should be made to leave at least a part of one ovary to keep up the internal secretion. The ovary should also be removed in operations for inflammatory disease when it is so extensively inflamed that a restoration to normal is apparently impossible. As a commentary to this last indication I would add that I believe that the more operations one does the less likely one is to remove ovaries through this indication.

In hysterectomy for myoma it is sometimes necessary to remove one or both ovaries on account of dense adhesions between the ovary and the growth. As a rule, one or both ovaries can and should be left.

Removal of normal ovaries on account of chronic pain "in the ovarian region," or double oëphorectomy in order to relieve dysmenorrhea is not indicated.

\section{HYSTERECTOMY.}

As in oöphorectomy, the more major operations one does the less inclined one is to do hysterectomy. The same general principles hold good here as in the preceding considerations. Removal of the normal uterus, because the woman has come vague "female trouble" which does not yield to treatment, is never justifiable. Clear, well-defined indications are always necessary to justify hysterectomy.

Such indications are given for the most part in malignant disease of the uterus or ovaries, myomata, pelvic inflammatory disease in which the uterus is generally involved or in which the process is tuberculous in character, some cases of complete prolapse and a few cases of intractable uterine hemorrhage arising from microscopic changes in the endometrium or uterine musculature.

\section{CONCLUSIONS.}

I will not carry this discussion of special operative procedures farther. It has been seen that there are certain considerations common to the discussion of all of them. I wish, in conclusion, to deduce from these common factors a few simple principles, the strict observance of which is of vital importance in the successful practice of elective surgery.

Before it can be said that any given operation is necessary or advisable three conditions must be satisfied beyond reasonable doubt:

First, we must be satisfied that the symptoms of which the patient complains are caused by the lesion toward which the operation is directed.
Second, we must have a reasonable assurance that these symptoms will be relieved entirely or greatly benefited by the operation which is proposed.

Finaily, we must be reasonably certain that the probable danger from the operation itself, when compared with the severity of the lesion and the expectation of relief, is small enough to justify the patient in taking the operative risk.

These postulates seem absurdly simple and self-evident. They are, of course, the fundamental principles of all therapeutics. But in elective surgery, more than in any other department of medicine, we are prone to lose sight of them or to minimize their importance. In just so much as we disregard them in the same proportion will the results of our surgical work fall below the high plane of excellence. If we should carefully consider them before every proposed operation of elective surgery I am sure that, while we might not do as many operations as we may be doing now, on the other hand, the end results of the operations which we would do would be much more brilliant than at present.

228 Hogan Street.

\section{EARLY AND LATE CASES OF GASTRIC ULCER.*}

WILLIAM FITCH CHENEY, M.D.

Professor of Principles and Practice of Medicine, Cooper Medical College, and Physician to Lane Hospital. SAN FRANCISCO.

Two conclusions have impressed themselves on me regarding gastric ulcer: 1, Heretofore the condition has not been diagnosed early enough; 2 , it has not received sufficiently rigorous treatment after the diagnosis has been made.

\section{NEED OF EARLY DIAGNOSIS.}

Early diagnosis is of immense value as an aid to cure, certainly as much so as in gastric cancer, possibly as much so as in pulmonary tuberculosis. It has been too much the custom in the past to consider no case gastric ulcer unless certain definite evidence was present, such as vomiting of blood, blood in the stools, violent attacks of gastralgia and lancinating pain through the abdomen from epigastrium to back. These signs are classical and are usually accepted as convincing, but diagnosis ought to be made and treatment instituted before this stage has been reached.

My own conviction is that many cases of chronic dyspepsia, characterized for weeks or months by flatulence, waterbrash, burning and distress after food, where the epigastrium shows tenderness and the test meal shows hyperacidity, are in reality gastric ulcer, even when the feces show no occult blood. Such cases can not positively be diagnosed as ulcer any more than incipient tuberculosis can positively be diagnosed before bacilli are found in the sputum; yet they deserve routine treatment on suspicion, in one instance as truly as in the other. If thesc dyspeptic cases are allowed to go on indefinitely without treatment or with only haphazard treatment, serious symptoms are likely sooner -or later to appear that make the diagnosis clear but that render the results of treatment much more dubious. I would not urge the ulcer cure at once in every case of acid dyspepsia, but if one month's treatment by ordinary methods, with the patient up and about, does not cause a marked decrease of the trouble I have learned to insist

\footnotetext{
* Read before the Callfornia Academy of Medicine, June, 1907.
} 